\title{
GENERATION OF DECELERATION PULSES OF THE TROLLEY IN CHILD RESTRAINT SYSTEMS' DYNAMIC TESTS BASED ON SIMULATIONS AND EXPERIMENTAL VALIDATIONS
}

\author{
ZHE WEI*1 \\ ${ }^{1}$ National Quality Supervision and Inspection Center for Engineering Machinery, China \\ Academy of Machinery Science and Technology. Daxing, Beijing, China
}

\begin{abstract}
As one of devices for passive safety, child restraint system remains a positive factor in securing the safety of child occupants in vehicles' collisions. Generation of deceleration pulses is essential for dynamic tests conducted to assess the safety performance according to many regulations such as UN Regulation No. 44. It deserves research on how to generate the qualified pulses conveniently and with low cost. A new method of convenience using steel pipes or tubes is recommended after all the factors influencing the deceleration being ascertained. Varied combinations of parameters make it possible and easy to optimize the deceleration pulses.
\end{abstract}

Keywords: deceleration pulse, child restraint system, dynamic test

\section{INTRODUCTION}

Child restraint systems are of great importance in securing the safety of child occupants in vehicle collisions, and as a kind of passive safety system, such devices should satisfy the relevant requirements of technical regulations and standards such as UN Regulation No. 44, UN Regulation No. 129, and GB 27887-2011, etc. [1]. Dynamic tests remain the major items of regulations and the most important means to simulate real crashes and to evaluate safety performance of child restraint systems. Deceleration pulses being the key factor that affects test results, can be generated by various methods including employing the trolley of acceleration type, using special materials and structures [2], etc. Among the methods, it's especially vital to devise or find a cheap and convenient one for the generation of deceleration pulses, because a lower cost makes it easy to inspect and verify devices for passive safety including but not limited to child restraint systems, thus bringing the advantages of flexibility and competition for smaller-scale laboratories and factories. This research focuses on how to achieve the purpose by using steel tubes and pipes that have been processed according to some treatment scheme. Easy availability and handling of different tubes or pipes should be taken into full consideration and utilized to militate in favor of conceiving approaches to the generation of deceleration pulses.

Actually, child restraint system's dynamic test is one of the most effective methods to evaluate its integral safety which is directly related with and has an effect on the function ensuring the ability to protect child occupants including newborn baby, infants, toddlers and other older children [3-6]. Many laboratories and factories have been using the acceleration-type trolley to carry out dynamic tests in which the acceleration/deceleration pulses can satisfy the requirements of regulations or standards very well with the use of the equipment. However, the acceleration-type trolley is quite expensive for smaller-scale laboratories and factories and has a complex structure, thus making it not so easy to maintain as the deceleration-type. Meanwhile, the deceleration-type trolley can have various means to absorb the kinetic energy and to generate deceleration pulses in the process of

\footnotetext{
* Corresponding author, email: wzhk2008@yeah.net

(C) 2021 Alma Mater Publishing House
} 
changing the state of motion and be much cheaper compared with the acceleration-type one as well. Therefore, the deceleration-type trolley is still used widely and popular with small laboratories and factories because of its flexibility, convenience and low cost.

The current research mainly explores the key technology of deceleration-type trolley, i.e. the method to produce deceleration pulses required in a crash test according to different standards. Deceleration pulses produced by the trolley simulate the actual pulses in a vehicle crash to some extent [7-9]. Generation of qualified pulses is important to evaluate child restraint system's dynamic properties and promote the standardization of the products, and is also an essential prerequisite for conducting dynamic tests according to regulations or standards strictly [10-12]. Generally, deceleration pulses are generated by various kinds of energy-absorbing mechanisms [13-15]. Energy-absorbing materials and structures can be applied to that use. The shape of the pulses depends upon and reflects the trolley's deceleration when energy-absorbing mechanism functions. Hence, the mechanism must be of great convenience and reliability to ensure conformity to regulations and efficiency of operation. The national standard of China, GB 27887-2011 specifies a kind of energy-absorbing mechanism, which functions when an olive-shaped knob compresses a polyurethane tube inserted into a steel tube on trolley. However, the knob sometimes gets jammed in the tube and different deceleration pulses need different pairs of knobs and tubes. Once specified, the pulses exclude all the other pairs of knobs and tubes except the corresponding ones. There are also other kinds of mechanisms used to absorb the trolley's kinetic energy and generate pulses in China, and they can include steel plates, steel bars and hydraulic devices, etc.

Considering that the quantity of steel plates and steel bars needed in dynamic tests appears large, and it's timeconsuming indeed to replace the used ones, the hydraulic devices have high failure rates as well, an alternative way to absorb the kinetic energy is therefore presented in the article, and it employs a kind of mechanism mainly consisting of a sliding block with 4 rectangular holes fixed on the trolley and 4 steel pipes with specified characteristics. The impact brought about by trolley is buffered when the process of sliding block squeezing the pipes begins. In view of the simplicity of its structure, this mechanism is convenient to adjust and control, thus bringing about easy manipulation of the state of trolley. By simple and effective means, to ascertain factors influencing deceleration pulses in child restraint systems' dynamic tests for trolleys of deceleration type, numerical simulations could be a good choice. Tests for experimental validation can be carried out to check the reliability of the simulation scheme [16]. After the validation, the main factors that include the parameters of pipes and trolley and could affect the shapes of deceleration pulses in dynamic tests can be ascertained and then undergo proper adjustments for further optimization. Furthermore, the deceleration curves have been specified in technical regulations, and could be perceived as the object to approach to. To a great extent, the deceleration pulses should coincide with the curves defined by relevant regulations. Therefore, ensuring the maximum coincidence inevitably involved optimization and reconfiguration, in view of the uncertainty and complexity of combining testing conditions and results with the simulation ones. All the factors influencing deceleration pulses in tests could be combined to or separately change the trends, peak values, width and other parameters of the pulses. Moreover, since the deceleration pulse in rear-impact test is much easier to generate than the frontalimpact test, the way to achieve the generation of deceleration pulses in frontal-impact test is regarded as the aim, and the emphasis is placed on ascertaining the relevant factors and optimizing the pulses.

The paper in the field of mechanical engineering seeks to highlight and analyze problems in the generation of deceleration pulses of the trolley in child restraint systems' dynamic tests and offers some flexible and effective proposals to solve them and to obtain the qualified pulses based on simulations and experiments.

\section{METHODS}

\subsection{Scheme of absorbing kinetic energy of trolley}

The velocity of trolley in child restraint system's frontal impact test is $48 \mathrm{~km} / \mathrm{h} \sim 50 \mathrm{~km} / \mathrm{h}$. Therefore, a proper energy-absorbing device should be used to buffer the impact of the moving trolley, and the qualified deceleration that meets regulations is achieved in the process. The deformation of steel pipes will absorb energy and make the trolley gradually decelerate. So, the following factors should be taken into consideration, i.e., the thickness, the slope, the heat treatment of steel pipes, the rectangular holes' sizes in the sliding block, the velocity, and the weight of trolley, etc., for they are related with the production and transfer of energy. All the factors are obviously major influences compared with other ones, for they are related most closely with the extent of pipes' deformation which absorbs most of the trolley's kinetic energy. A special mechanism used as the energyabsorbing device was designed, as illustrated in Figure 1. Before a dynamic test, the fixture, child restraint 
system and child dummy were installed on the trolley according to relevant regulations, as displayed in Figure 2, and after installation the trolley was ready for a pull force to tow it to a specific position from which it would then be released and accelerate to the specified velocity. As shown in Figure 3, steel pipes were installed on the trolley for a test, and the deformation would begin when the brake rods came into contact with the barrier.

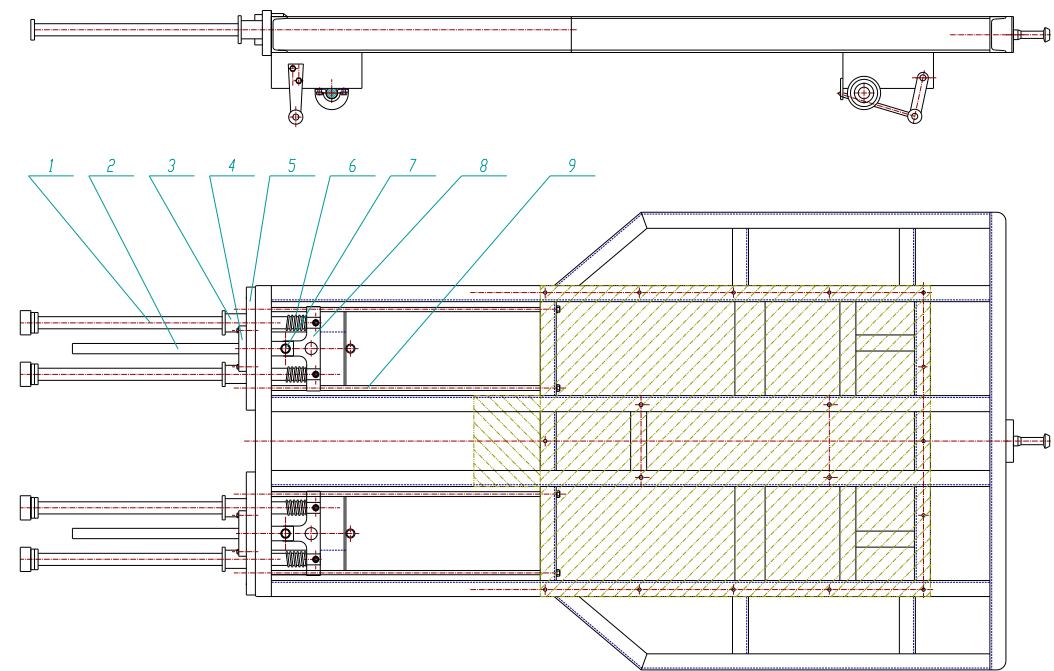

Fig. 1. Schematic diagram of trolley's structure:

1 - brake rods; 2 - steel pipes; 3 - guiding cylinder; 4 - sliding block; 5 - panel; 6 - spring; 7 - bolt; 8 - connecting block; 9 - guiding rod.

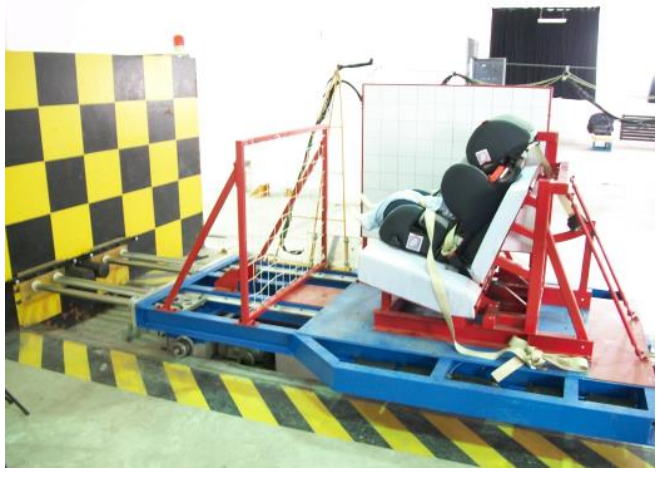

Fig. 2. The trolley after installation.

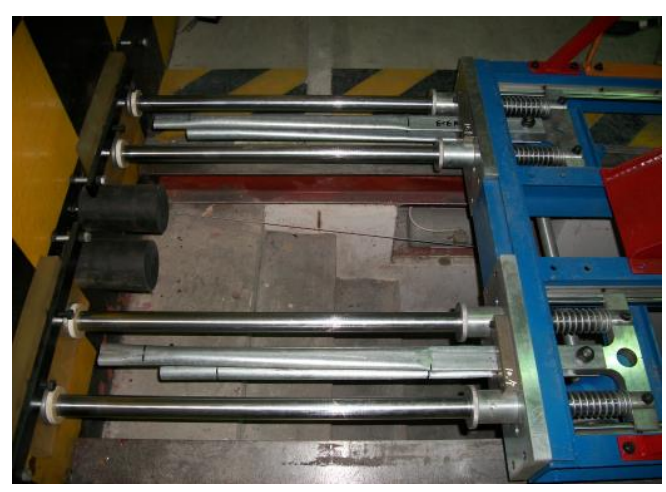

Fig. 3. Energy-absorbing mechanism.

\subsection{Simulation scheme}

Proe-Ansys-Lsdyna combined modelling and solving method was adopted to conduct simulations, as shown in Figure 4. The software Proe was used to construct 3D-models of steel pipes. The keyword files were created by Ansys after meshing the model, defining material properties, boundary conditions, and the loads, etc. Then the keyword files were edited manually and loaded to the solver to meet the requirements for altering the parameters of pipes and the trolley. Lsdyna solver was used for calculations and Ls-PREPOST finished the post process. With the means could different types of crash tests be simulated without omitting any necessary setups or conditions, and the cost was kept low enough compared with dynamic tests.

In order, to validate the reliability of this simulation scheme, the initial conditions were set at will within the proper range in both of the simulation and test, i.e., the conditions in the simulation were the same as the test ones. After numerical simulation and real test, results were compared between them, as reflected in Figure 5, the tendencies of simulations and test's deceleration pulses were the same as each other after filtration using CFC 60 filter, although they did not coincide at some pairs of points. Despite the fact that the tendencies of pulses in simulation and in test resembling each other, the ideal model of simulation would unavoidably be affected by its limitations because of the complexity of test conditions, i.e., simulation results could not be identical to test ones even for tiny changes in test conditions. However, the accuracy of simulation and its deceleration pulse's 
coincidence with the test results was enough for the use mentioned in the current research. Hence, the simulation scheme was reliable and could be used to carry out the following tasks.

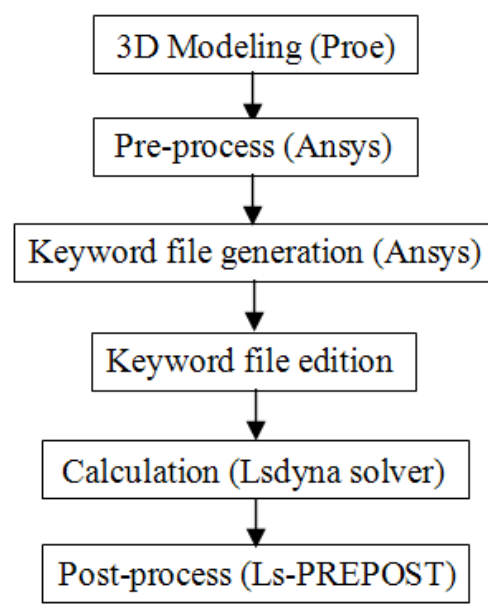

Fig. 4. Flow chart of simulation.

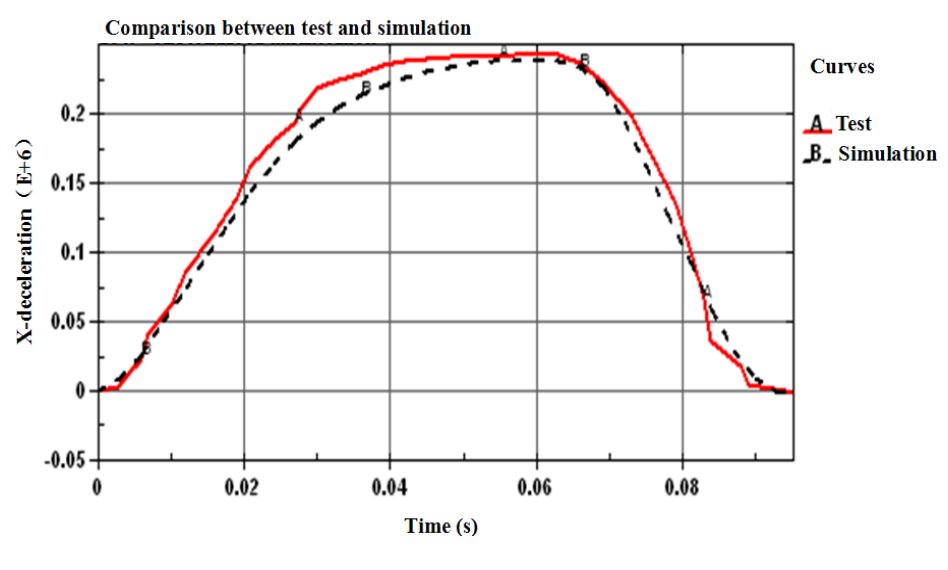

Fig. 5. Comparison between test and simulation.

\subsection{Ascertaining the relevant factors and optimization}

Since the effect of different parameters upon deceleration pulses' shapes was focused on, all the relevant parameters' values were changed in accordance with certain rules. Simulations for ascertaining the factors were simplified so as to ensure the accuracy and universality, with the fact taken into account that the structure of the trolley mentioned above to absorb kinetic energy consisted of 4 steel pipes and other related parts, and when collisions happened, only the sliding block's rectangular holes squeezed the pipes to deformation, and simulation process could be perceived as the sliding block with one fourth of the trolley's total weight squeezing 1 pipe. That was effective and time-saving in calculations and ascertaining which parameter took effect and how the shapes of deceleration pulses were influenced, although a combination of 2 pairs of pipes was employed in actual tests. Finally, all the pulses underwent filtration by CFC 60 Butterworth IV filter. By comparing the results of simulations and the trends of pulses, it would be clear how the parameters mentioned above affected the energy-absorbing mode and exerted influence on deceleration pulses. Different combinations of the factors could then be made full use of to optimize the shapes, the trends and other parameters of deceleration pulses.

\section{RESULTS AND DISCUSSION}

\subsection{Factors related with steel pipes}

Slope angles of the end of steel pipes, the thickness, the diameter, and the heat treatment of the pipes being 4 main factors that affect deceleration, they are related directly with the pipes. Although the diameter is a significant factor that can affect the deceleration pulses' shapes greatly, it is not suitable for controlling the pulse and not used as the means to adjust the pulses in dynamic tests, for sliding block's structure determines that the rectangular opening's height can only achieve the installations of pipes of certain diameters exclusively. Besides, steel pipes have been standardized, thus making the size of sliding block's rectangular hole within a specific range. Once the diameters of pipes determined, the basic shapes of deceleration pulses could be formed. When the diameters undergo changes, the deceleration will vary substantially and even exceed the proper range. So, it's meaningless to select the diameters as the way to control the deceleration especially if slight adjustment becomes necessary. Moreover, the rectangular hole's size, i.e. the height of the opening is hard or impossible to adjust after the sliding block is made. So only the other three factors are taken into consideration.

As to the slope, as shown in Figure 6, it can be concluded that the bigger the slope angle is, the sooner the peak value reaches. As is revealed in Figure 7, simulation results show that the thickness has close relation with pulse width, peak value and time to peak, indicating negative correlation, positive correlation and negative correlation respectively. In Figure 8, it is illustrated that heat treatment of pipes also influences deceleration pulses, for heat treatment causes changes of the material's yield stress which is of great importance in resisting deformation. Relations between yield stress values and deceleration pulses show that the factor has almost the same effect as the thickness on deceleration of trolley. As a matter of fact, it is a priority to choose thickness between the two as 
the major factor for pulse control, for it's easier and more convenient to deal with. Meanwhile, the availability of different series of pipe thickness and the difficulty of controlling the yield stress accurately by heat treatment lead to the fact that a perfect scheme of deceleration control should take pipe thickness into consideration.

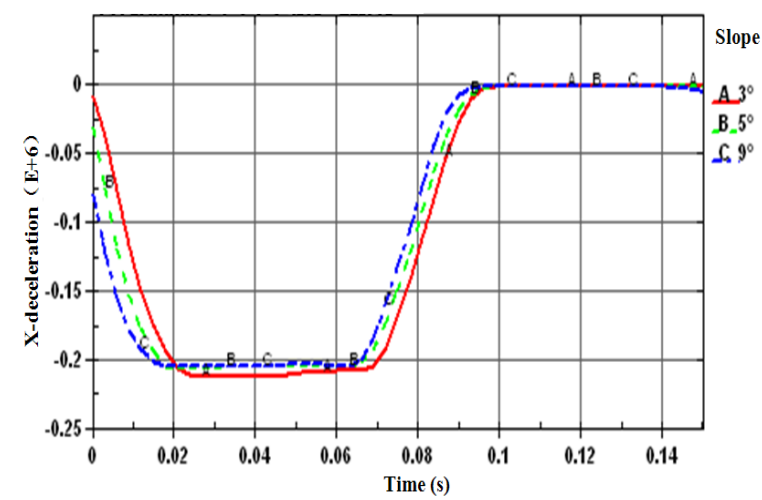

Fig. 6. Effect of pipe's slope.

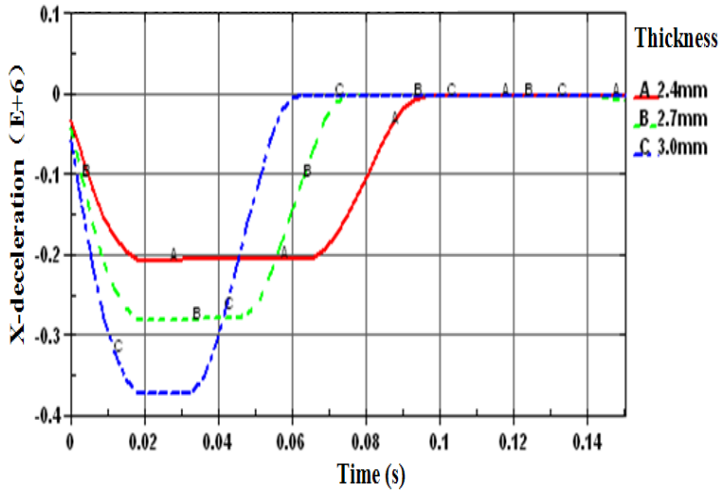

Fig. 7. Effect of pipe's thickness.

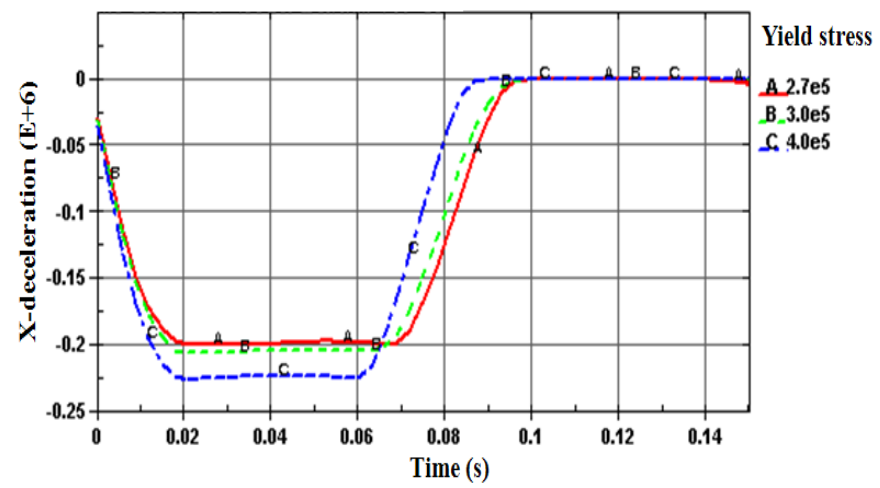

Fig. 8. Effect of yield stress.

\subsection{Factors related with the trolley}

Factors related with trolley mainly include sliding block's opening height, trolley's velocity and its weight. Results in Figure 9 make it clear that the opening height has the opposite effect on deceleration pulses with pipes' thickness and yield stress, i.e., it shows a completely different pattern with those mentioned above. As indicated in Figure 10, the pulse width increases with trolley's velocity. But the velocity has no relation with the pulse's peak value and the time to peak. It can be inferred that the trolley weight plays the same role as the opening height does according to the information in Figure 11. The increase of trolley's weight results in the decrease of pulse's peak value in dynamic tests. But there exists positive correlation between the pulse width and time to peak with the weight of trolley.

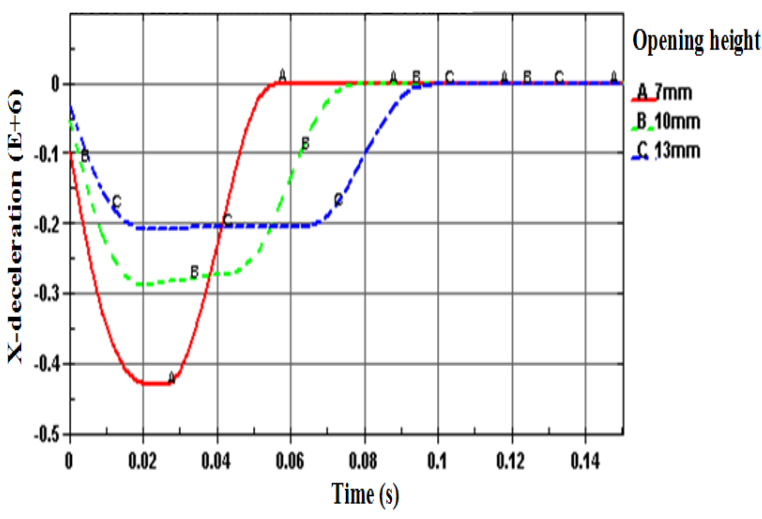

Fig. 9. Effect of the height of sliding block's opening.

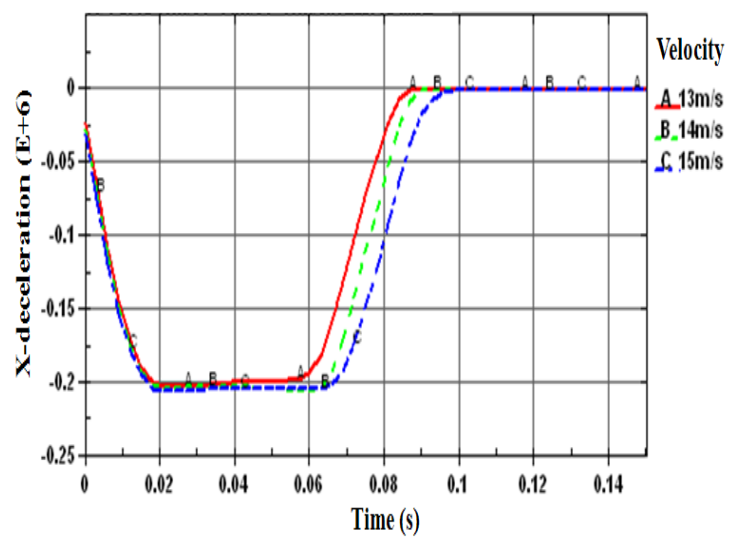

Fig. 10. Effect of trolley velocity. 


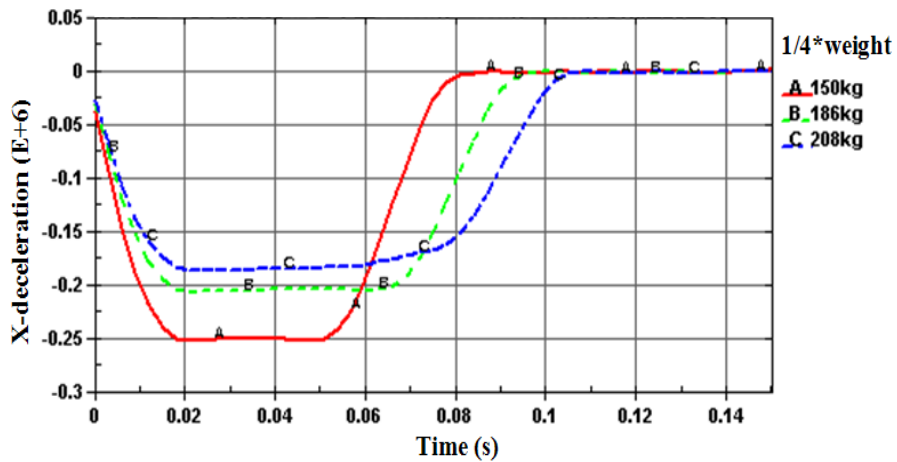

Fig. 11. Effect of trolley's weight.

\subsection{Summary of simulation results}

As tabulated in Table 1, simulation results display 2 different correlations including positive and negative ones between the factors and the pulses. Theoretically, different kinds of deceleration pulses can be generated by combining the factors properly. Even the quantity of steel pipes can be added or subtracted to satisfy different requirements. Therefore, the adjustment is more flexible to a great extent compared with other kinds of energyabsorbing mechanisms.

In Table 1, the symbols "×", “+ ", “ - " represent no correlation, positive correlation and negative correlation respectively. Positive correlation is a relationship between two variables in which both variables move in tandem i.e., in the same direction. A positive correlation exists when one variable decreases as the other variable decreases, or one variable increases while the other increases. Negative correlation is a relationship between two variables in which one variable increases as the other decreases, and vice versa.

Table 1. Summary of simulation results.

\begin{tabular}{|c|c|c|c|c|c|c|}
\hline \multirow{2}{*}{ Parameters } & \multicolumn{4}{|c|}{ Pipe } & \multicolumn{3}{c|}{ Trolley } \\
\cline { 2 - 7 } & Slope & Thickness & Yield stress & Opening height & Velocity & Weight \\
\hline pulse width & $\times$ & - & - & + & + & + \\
\hline peak value & $\times$ & + & + & - & $\times$ & - \\
\hline time to peak & + & - & - & + & $\times$ & + \\
\hline
\end{tabular}

\subsection{Optimization of deceleration pulses}

In order, to simulate dynamic tests more accurately, another 3 simulations under actual conditions listed in Table 2 were conducted. As shown in Table 2, in the 3 simulations, pipes' thickness, quantity, and the trolley's weight, opening height and velocity were all the same as one another. No pipes underwent heat treatment. The sole differences lay in the slope angles of one or two ends of the pipes.

Table 2. Simulation layout.

\begin{tabular}{|c|c|c|c|c|c|c|c|c|}
\hline No. & Head slope & End slope & Thickness & $\begin{array}{c}\text { Heat } \\
\text { treatment }\end{array}$ & $\begin{array}{c}\text { Quantity } \\
\text { of pipes }\end{array}$ & $\begin{array}{c}\text { Opening } \\
\text { height }\end{array}$ & $\begin{array}{c}\text { Trolley } \\
\text { velocity }\end{array}$ & $\begin{array}{c}\text { Trolley } \\
\text { weight }\end{array}$ \\
\hline 1 & $2^{\circ} \& 5^{\circ}$ & $0^{\circ} \& 0^{\circ}$ & $2.5 \mathrm{~mm}$ & no & 2 pairs $(4)$ & $12 \mathrm{~mm}$ & $49.7 \mathrm{~km} / \mathrm{h}$ & $800 \mathrm{~kg}$ \\
\hline 2 & $1.2^{\circ} \& 2^{\circ}$ & $0^{\circ} \& 0^{\circ}$ & $2.5 \mathrm{~mm}$ & no & 2 pairs $(4)$ & $12 \mathrm{~mm}$ & $49.7 \mathrm{~km} / \mathrm{h}$ & $800 \mathrm{~kg}$ \\
\hline 3 & $1.2^{\circ} \& 2^{\circ}$ & $0^{\circ} \& 2^{\circ}$ & $2.5 \mathrm{~mm}$ & no & 2 pairs $(4)$ & $12 \mathrm{~mm}$ & $49.7 \mathrm{~km} / \mathrm{h}$ & $800 \mathrm{~kg}$ \\
\hline
\end{tabular}

In the first simulation, 4 pipes were installed onto the sliding block whose rectangular hole would squeeze all the pipes to full deformation. A pair of pipes consisted of 2 identical ones, and the 2 pairs' head slope angles were $2^{\circ}$ and $5^{\circ}$ respectively. As is shown in Figure 12, the time to peak is not satisfying and peak value arrives too early. In order, to solve the problem, the head slope angle could be decreased properly. In the second simulation, 4 pipes were still used and 2 of them had $2^{\circ}$ head slope angles instead of $5^{\circ}$, while the others had $1.2^{\circ}$ head slope angles. The end slope angles remained unchanged, and were still $0^{\circ}$. The result reveals that the latter pulse is better than the former one, on the basis of information in Figure 12. But the end of the latter pulse appears a little steep and easy to be out of boundary if some disturbances occur. Hence, it can be optimized further. Squeezing the pipes in advance to make them have specific slopes is a cheap way to adjust the pulses and it's easy to achieve. So, the end slopes of 1 pair were processed, and 2 pipes had $1.2^{\circ}$ head slope angles and $0^{\circ}$ end slope 
angles, the other 2 pipes had $2^{\circ}$ head slope angles and $2^{\circ}$ end slope angles. The third simulation result shows that this pulse satisfies the requirements of ECE R44 and GB 27887-2011, etc., and its shape is better than the other two.

The deceleration pulses in Figure 13 were generated in another actual dynamic test and in the corresponding simulation, and conditions in the simulation and in dynamic test were almost the same as each other. Comparison between this real pulse and the one obtained by simulation, further validates that the simulation scheme is reliable and of great use to provide a solution. Furthermore, the energy-absorbing mechanism proves to be effective in generation of deceleration pulses.

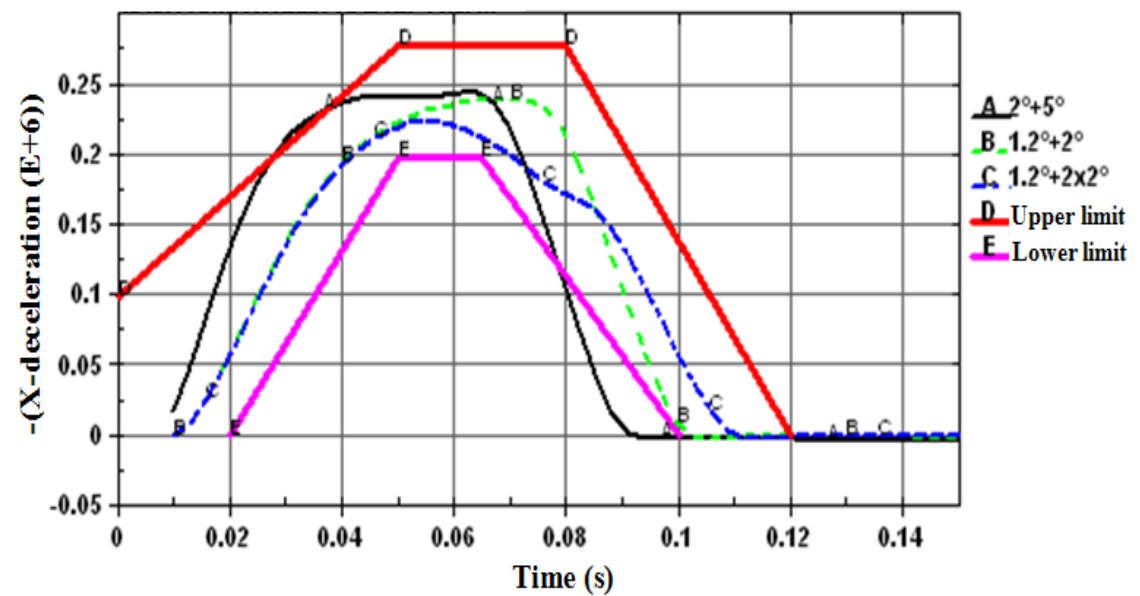

Fig. 12. Simulation results under 3 different conditions.

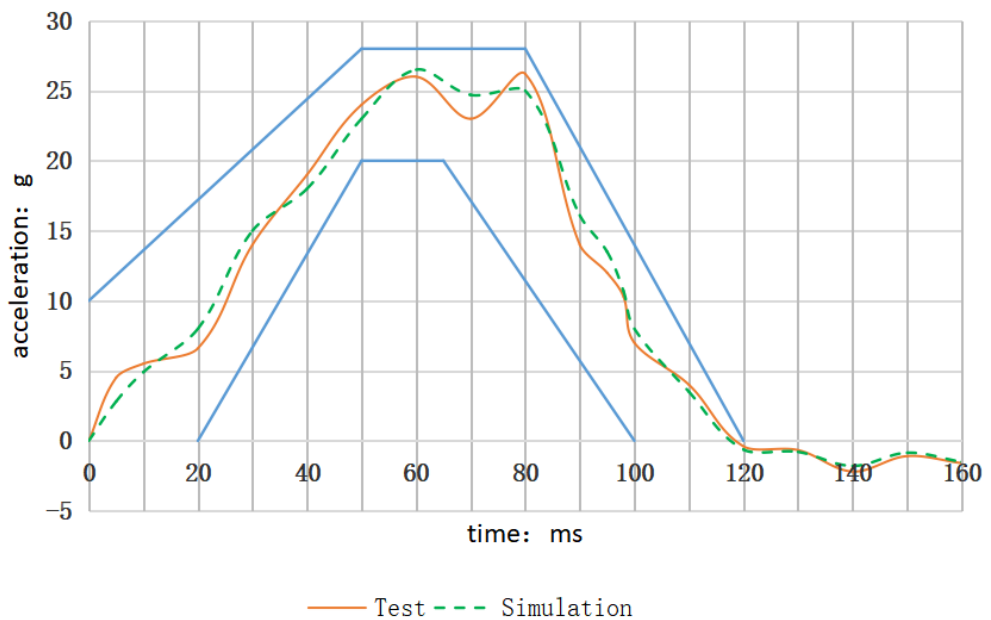

Fig. 13. Comparison between test and simulation.

\section{CONCLUSIONS}

A kind of energy-absorbing mechanism used to generate deceleration pulses for deceleration type trolley in child restraint systems' dynamic tests was designed. Factors affecting the deceleration were ascertained with the means of simulations and validation tests. The adjustment of deceleration pulses by changing the parameters or combining the factors proves to be effective and efficient, thus making it possible and easy for further optimization. Other deceleration pulses in rear-impact tests can also be generated in the same way. Moreover, this research minimized the quantity of tests and reduced the cost greatly by simulations, with dynamic tests having validated that the simulation results were reliable. It can be said that the aim of generation and optimization of deceleration pulses has been achieved. Further researches can be conducted on the assessment of child restraint system's safety performance, the injury mechanism of child occupants, etc., based upon the qualified deceleration pulses. 


\section{REFERENCES}

[1] Kapoor, T., Altenhof, W., Snowdon, A., Howard, A., Rasico, J., Zhu, F.C., Baggio, D., A numerical investigation into the effect of CRS misuse on the injury potential of children in frontal and side impact crashes, Accident Analysis and Prevention, vol. 43, no. 4, 2011, p. 1438 - 1450.

[2] Peng, Y., Deng, W., Xu, P., Yao, S., Study on the collision performance of a composite energy-absorbing structure for subway vehicles, Thin-Walled Structures, vol. 94, 2015, p. $663-672$.

[3] Nazif-Muñoz, J.I., Nandi, A., Casares, M.R., Impact of child restraint policies on child occupant fatalities and injuries in Chile and its regions: An interrupted time-series study, Accident Analysis and Prevention, vol. 120, 2018 , p. $38-45$.

[4] Sivasankari, S., Balasubramanian, V., Influence of occupant collision state parameters on the lumbar spinal injury during frontal crash, Journal of Advanced Research, vol. 28, 2021, p. 17 - 26.

[5] Razaghi, R., Biglari, H., Hasani, M., Karimi, A., Comparative numerical study on the child head injury under different child safety seat angles, Theoretical and Applied Mechanics Letters, vol. 9, no. 4, 2019, p. 260 - 263.

[6] Baranowski, P., Damaziak, K., Malachowski, J., Mazurkiewicz, L., Muszyński, A., A child seat numerical model validation in the static and dynamic work conditions, Archives of Civil and Mechanical Engineering, vol. 15 , no. 2 , 2015, p. $361-375$.

[7] Li, Z., Ge, H., Zhang, J., Zhu, Y., The necessity of evaluating child neck injury in frontal collision of school bus for transportation safety, Safety Science, vol. 62, 2014, p. $441-449$.

[8] Ha, D.R., Bertocci, G., Injury risk of a 6-year-old wheelchair-seated occupant in a frontal motor vehicle impact - 'ANSI/RESNA WC-19' sled testing analysis, Medical Engineering and Physics, vol. 29, no. 7, 2007, p. $729-738$.

[9] Beeman, S.M., Kemper, A.R., Madigan, M.L., Franck, C.T., Loftus, S.C., Occupant kinematics in low-speed frontal sled tests: human volunteers, hybrid III ATD, and PMHS, Accident Analysis and Prevention, vol. 47, 2012, p. 128 - 139.

[10] Karimi, H.R., Pawlus, W., Robbersmyr, K.G., Signal reconstruction, modeling and simulation of a vehicle full-scale crash test based on Morlet wavelets, Neurocomputing, vol. 93, 2012, p. 88 - 99.

[11] Li, N., Fang, H., Zhang, C., Gutowski, M., Palta, E., Wang, Q., A numerical study of occupant responses and injuries in vehicular crashes into roadside barriers based on finite element simulations, Advances in Engineering Software, vol. 90, 2015, p. $22-40$.

[12] Brolin, K., Stockman, I., Andersson, M., Bohman, K., Gras, L.L., Jakobsson, L., Safety of children in cars: A review of biomechanical aspects and human body models, IATSS Research, vol. 38, no. 2, 2015, p. 92 - 102.

[13] Li, Q.Q., Li, E., Chen, T., Wu, L., Wang, G.Q., He, Z.C., Improve the frontal crashworthiness of vehicle through the design of front rail, Thin-Walled Structures, vol. 162, no. e107588, 2021.

[14] Abdulqadir, S.F., Design a new energy absorber longitudinal member and compare with S-shaped design to enhance the energy absorption capability, Alexandria Engineering Journal, vol. 57, no. 4, 2018, p. 3405 - 3418.

[15] López, D.A., Lozano, M.S., Sáez, L.M., Frontal crashworthiness characterisation of a vehicle segment using curve comparison metrics, Accident Analysis and Prevention, vol. 117, 2018, p. 136 - 144.

[16] Ciampaglia, A., Fiumarella, D., Niutta, C.B., Ciardiello, R., Belingardi, G., Impact response of an origamishaped composite crash box: Experimental analysis and numerical optimization, Composite Structures, vol. 256, no. e113093, 2021. 